\title{
Internet of Things Technology-Based Manufacturing Inventory Management
}

\author{
Hanbin Wang \\ School of Business and Information Technology \\ Quanzhou Normal University \\ Quanzhou, China \\ wanghanbin@qztc.edu.cn
}

\author{
Xiaoxia Chen \\ School of Business and Information Technology \\ Quanzhou Normal University \\ Quanzhou, China \\ 649047197@qq.com
}

\begin{abstract}
As the emerging information network, more and more attention are paid to the internet of Things technology. For the application in the field of logistics, tracking of goods and information traceability provides advanced technical support. In recent years, China's manufacturing industry rise rapidly, but the level of automation of inventory management, safety stock, the collection of information is not accurate. Things technology has the advantage of better information traceability, data processing and other aspects. The data acquisition in the manufacturing process of inventory management can help to solve the above risks. Articles from the status of Manufacturing Inventory Management, its existence doesn't update timely. The page builds a manufacturing inventory management model based on Internet of Things technology, and also explains the design and implementation of the model. Finally it proposed measures to promote the manufacturing inventory management model based on Internet of Things technology.
\end{abstract}

Keywords-internet of things; inventory management; demand forecasting; inventory control

\section{MANUFACTURING INVENTORY MANAGEMENT STATUS} QUO

A. Inventory Management, Mechanization And Automation Level Are Low

According to incomplete statistics, China has built a three-dimensional warehouse which has more than 300 seats, and more than 50 fully automatic three-dimensional warehouses. On the one hand, the mechanization and automation of the warehouse inventory management levels is still relatively low; On the other hand, it have been completed and three-dimensional warehouse is in low utilization. The effect is not obvious. Advantage is not prominent, many library field resources are idle, and especially some small batch and single production enterprises warehouse automation. The library field facilities and equipment resources become more prominent. To enhance the effectiveness in the field of logistics, we must attach great importance to the mechanization and automation of warehouse management and effectiveness.

\section{B. Business inventories are generally higher, take up a lot of money}

China's manufacturing inventories remain at a high level in 2012. It can be seen from the current year PMI. The enterprise warehouse inventory is generally higher. , Companies have to ensure continuity of production and operation must save a certain amount of inventory, for many small and medium-sized enterprises. In the same time, the goods are bound to happen storage costs and inventory custody fees. That is more required to pay the storage costs and custody fees are the higher [1].

TABLE I. 2012 CHINA'S MANUFACTURING PMI SUB-INDEX (UNIT :\%)

\begin{tabular}{|l|c|c|c|c|c|c|c|c|c|c|}
\hline \multicolumn{1}{|c|}{ Month } & January & February & March & April & May & June & July & August & September & October \\
\hline Produce & 53.6 & 53.8 & 55.2 & 57.2 & 52.9 & 52.0 & 51.8 & 59.0 & 51.3 & 52.1 \\
\hline New orders & 50.4 & 51.0 & 55.1 & 54.5 & 49.8 & 49.2 & 49.0 & 48.7 & 49.8 & 50.4 \\
\hline Raw materials inventory & 49.7 & 48.8 & 49.5 & 48.5 & 45.1 & 48.2 & 48.5 & 45.1 & 47.0 & 47.3 \\
\hline Employees & 47.1 & 49.5 & 51.0 & 51.0 & 50.5 & 49.7 & 49.5 & 49.1 & 48.9 & 49.2 \\
\hline Supplier delivery time & 49.7 & 50.3 & 48.9 & 49.6 & 49.0 & 49.1 & 49.0 & 50.0 & 49.5 & 50.1 \\
\hline \multicolumn{1}{c}{ PMI } & 50.5 & 51.0 & 53.1 & 53.3 & 50.4 & 50.2 & 50.1 & 49.2 & 49.8 & 50.2 \\
\hline
\end{tabular}

Source: China Statistical Information Network of PMI data are compiled of the first 10 months in 2012
Note: PMI = production $25 \%+$ orders $30 \%+$ inventory $10 \%+$ employees x $20 \%+15 \%$ distribution 
The table above shows the past nine months in the manufacturing purchasing manager's index almost around $50 \%$ of the critical point fluctuations, and a downward trend in the second half of the year. In view of raw materials, raw materials inventory index in September was $47.0 \%$. It is located below the critical point, indicating that the manufacturing raw materials inventory continue to decrease, but fell significantly narrowed. Inventory coefficient can also reflect the level of inventory. Inventory coefficient is between 0.8 and 1.2 , reflecting the inventory in a reasonable range; the inventory coefficient $>1.5$, reflecting the inventory of the alert level; the inventory coefficient $>2.5$, reflecting the inventory is too high. Operating pressures and risks are very large.

\section{Low degree of inventory management information}

Chinese SMEs Development Report (2011) shows that there are a considerable number of small and mediumsized enterprises of information technology to promote enterprise development. The policy of government support for private enterprise normalization construction is poorly understood. The survey data show that $80 \%$ of SMEs have the ability to access the Internet, but only $44.2 \%$ of the companies are used in the Internet business operations; less than $10 \%$ of SMEs in the implementation of ecommerce. On the one hand, due to the low level of enterprise information, the managers often depend on inventory management and decision-making based on experience; On the other hand, as the low level of information, the majority of small and medium-sized manufacturing enterprises often account inadequately. It is very difficult for inventory managers to timely complete understanding of the stock. The manufacturing enterprises are business-to-consumption of raw materials. Companies take a more traditional management, material storage and centralized billing requisitioned by artificial [2].

\section{MANAGEMENT AND ANALYSIS OF MANUFACTURING INVENTORY}

\section{A. data update is not timely, accurate rate is not high}

The traditional inventory management method, such as data update is not timely. This can be summarized in two aspects: first, inventory confusion, the goods out of storage or the shift library is not recorded in a timely manner. Due to the low level of business automation, many production operations can not be mechanized, mostly done by hand. Error-prone manual work, production data flow between employees lead to data loss, defacement, data distortion, etc easily. These risks are ultimately resulting in denied access to the real basis of reliable data and information. Information update speed is slow. It does not allow managers real-time tracking of warehouse operations, to grasp the production process of its decision-making.

\section{B. unreasonable demand forecast}

The production plan of the MRP system is arranged in forecasting demand and material availability. Once the plan has been formed, every process will be to promote the flow of raw materials or semi-finished products to the next production program. It must predict customer demand and estimate delivery time [4]. When suppliers receive orders further to its channels, for the same reason, it will further enlarge the number of ordering. Wrong predictions and projections will result in large quantities of inventory. Therefore demand forecast is extremely unreasonable.

\section{C. the amount of safety stock}

The safety stock mainly depends on the number of time from order to delivery interval, the interval longer the supply chain, the greater the orders fluctuations. The ordering time is very long, such as a week, bi-weekly, a month or even longer. The same time, there is a huge difference between dealers who often take the vehicle ordering and transport, due to the vehicle freight rates and the scattered freight rates. MRP It is necessary to avoid the impact of various uncertainties, such as natural disasters, personnel fluctuations, incidents. It must maintain a certain level of safety stock. There will be a certain amount of protection to consider. The root cause for this situation is that companies do not have reliable data and information. As the reality of volatility and uncertainty caused by the higher psychological preparedness of the enterprise, they had to deal with high inventory levels. We do not know that is high or low future demand. Excessive safety stock will take up a lot of liquidity and may hide a lot of management problems [5].

\section{BUILD THE INTERNET OF THINGS TECHNOLOGY- BASED MANUFACTURING INVENTORY MANAGEMENT MODEL}

A. Internet of Things technology obtains data acquisition quickly and reliably. There is a good prospect in Manufacturing Inventory Management

In recent years, some shoes and apparel companies have tested the water of Things, IOT technology into production, logistics management.[6] This electronic chip can help enterprises in the pipeline between stores, companies and manufacturers to set up a fast-running, the store sales information and inventory together effectively. Same time, through the electronic tags, the staff spends more time on customer service, which led to the growth in sales volume.

As the simple operation of RFID and a heavy workload, logistics management has been a growing body of research and application. Inventory management ensures accurate inventory information. [7] Internet of Things is driven by many of the traditional industrial structure adjustment and industrial upgrading; the final will also promote the development of the entire economic structure of the mode from extensive to intensive.

\section{B. Inventory Management Model Based On Internet Of Things Technology Design}

MRP inventory management methods, for example, the combination of the Internet of Things are a common technique in the field of logistics, especially its core 
technology of RFID and network technology. Inventory management model is based on Internet of things technology to build the basic flow to improve inventory management. The perception layer is related to the Internet of Things technology anytime, anywhere and access to items from the storage. The library plays an important part of the real data. All data and information eventually focused on inventory management center database.

C. Based On The Inventory Management Model Of The Internet Of Things Technology Design. The Internet of Things technology is applied to one of the main storage, a library, returns processing and inventory all inspectors' four links. Its function is designed as follows:

1) production planning design and implementation . According to market forecasts and customer contracts production planning, and then automatically calculated based on customer demand for finished parts, components and raw materials demand projections, according to the date of delivery of the finished product all parts of the production schedule and materials procurement schedule. First, the master production schedule, product structure and bill of materials, existing inventory input MRP system [8]. MRP system according to the master production schedule under the final demand and product structure information, the demand for products are based on the specific operating procedures broken down into the production planning. Finally, demand time is limit for placing orders to suppliers.

2) the storage management function Design and Implementation. Interpretation of the data is connected to the information center. RFID Goods implantation RFID tag record their own products, namely, electronic tag encoding. Still relatively due to the cost of the RFID tags, RFID tags installed on the outer carton or pallet [9]. Each completed a process; the manufacturing sector will add the name of the production process in the RFID tag, time, place, person in charge, the completion of production information. The content of the RFID tag is rich with the degree of completion. Each process is complete record of its materials as well as the amount of material and the degree of completion, each material or component inventory and the number of finished products can be easily retrieved from the database.

3) Returns processing Design and Implementation. From raw materials into the library, each unit of goods has unique electronic tags. Electronic tags will be accompanied from the raw materials into semi-finished products. In the production process, material damage, moisture contamination needs to return unforeseen circumstances. The purchasing department is responsible for.

4) Inventory verification Design and Implementation. Inventory by workers handheld reader to scan and identify each label, Get EPC (Electronic Product Code) on the pins in the real-time data transmission to the host computer system of the stock management center through the wireless data communication method [10], the host computer system summary data, filter, verify, generate statistics, automatic inventory for a short period of time three-dimensional warehouse, and the results of the analysis. Inventory is about to do a comprehensive statistical RFID tag scan data and the data obtained can do a ring with the past experience and historical data support analysis. Demand forecasting, inventory is lower than or close to the safety stock red line. The system should call the police. This link is to update the inventory data protection.

5) design and implementation of a database management. The MRP system according to the customer and order information, develop a delivery plan and notify the warehouse. The warehouse will be determined in accordance with the delivery plan library finished the type, quantity and time [11]. After the completion of the library scheduling, it will be finished by the library channel through the top of the RFID. To ensure that the acquired data storage items with RFID reader agreement, the storage door ground-mounted sensor scales can be calculated before storage.

\section{MEASURES TO PROMOTE THE INTERNET OF THINGS TECHNOLOGY-BASED INVENTORY MANAGEMENT MODEL}

\section{A. Increase In Storage Level Of Mechanization And Automation}

The use of the Internet of Things technology relies heavily on machinery and equipment, automated guided trucks, stackers, sorters, forklifts, tractors, conveyor belts and other warehouse machinery.[12]It is worth mentioning that three-dimensional warehouse mechanization and automation of typical signs the emergence of the automated warehouse. Automated warehouse compared to traditional warehouse to maximize the use of space as possible to meet production requirements, reduce labor intensity, reduce the rate of material consumption, improve production efficiency, to strengthen the production and materials management, building inventory backlog of funds, driven by computer technology, communication technology and information technology, information into in order to automate the development trend of threedimensional warehouse.

B. Unified Network And Device Interfaces, Protocols, Network Standardization To Achieve Material

Internet of Things is a multi-device, multi-network, multi-application, interoperability, and integration between network, including a variety of sensors, computers and communication networks. In combination with the inventory management system to these technologies, devices and systems connected a unified standard for the 
development of interface design. Communication protocol is particularly important. Standard interface will not be able to achieve the sharing of resources. In April 2012, the International Telecommunication Union examined the draft standard submitted by the country "Overview of Things. This standard covers the concepts, terminology, and technical view, characteristic of Things the basic content of the demand, the reference model, business model, reflecting the interest demands of our country in the networking industry.

\section{To Promote The Internet Of Things Technology In The Supply Chain}

Inventory management is a part of the supply chain. The efficient operation of a certain degree of Things is related to supply chain fluency. Internet of Things technology industry throughout the logistics supply chain, real-time visualization monitoring and management. In turn, efficient inventory management can help supply chain more quickly axis move, so closely related to the needs of both the Internet of things such binders and lubricants. There is a very wide field of application of things, and a strong cross-cutting nature of many industries, effective links in various industries, strengthen coordination and interaction between the industry. The formation of a unified Internet of Things technology-based inventory management, this mode is more universal, and can also get considerable economies of scale. Such as the textile industry and clothing industry, there is a big common. The two co-feasibility of the implementation of this management model is relatively large. Applied to the higher costs of manufacturing inventory management, networking, co-operation between the industries can integrate resources.

\section{ACKNOWLEDGMENT}

This research was supported by the funds from: Foundation item 1: Fujian university key project construction service routines "A Study on the Development of Regional Economies in Southern Fujian Province ", Project Number: B054; Foundation item 2: Science Plan Project of Quanzhou, Project Number: 2012Z109, 2012Z124; Foundation item 3: Science Plan Project of Fujian "Research of cycle development mode of Fujian agricultural processing industry - Quanzhou for Example".

\section{REFERENCES}

[1] Jinhan Xin, Wang Liang, Huo Yan warehousing and inventory management $[\mathrm{M}]$. Chongqing: Chongqing University Press, 2008,106. (in Chinese)

[2] Dimon modern enterprise inventory management method discussed [J] Science and Technology Information, 2009 (33): 105. (in Chinese)

[3] Yang Xuan. Material requirements planning MRP analysis and design [D] master's degree thesis, Shandong University, 2009. (in Chinese)

[4] Zeng Sen Yao of Things, inventory management and RFID [J]. TRADE INDUSTRY, 2010 (17): 84. (in Chinese)

[5] Serve for Guanyu Wang Jiao Yuntao Things technology in the field of modern logistics [J]. Automation Technology and Application, 2011 (10): 34. (in Chinese)

[6] Christopher Cheng manufacturing enterprise inventory management status quo with modern business [J], 2009 (02): 84. (in Chinese)

[7] Summer months. BASF dispersion departments imported products inventory management problem [D] a master's degree thesis, Shanghai Jiao Tong University, 2009. (in Chinese)

[8] Tang forwards the development status and trends of the Internet of Things Industry [J]. Industry, 2010 (6): 20. (in Chinese)

[9] Zhang Duo of Things trend [M] Beijing: Tsinghua University Press, 2010,34 (in Chinese)

[10] Song Yajie of Things technology-based inventory control [J] Commercial Economic Research, 2010 (24): 35. (in Chinese)

[11] Yong Kim, warehouse management practices [M]. Hangzhou: Zhejiang University Press, 2008,136. (in Chinese)

[12] Wei Zhen-Lin, the Jishou Wen, Wong Ain-ling. Logistics, information technology and equipment [M] Beijing: China Material Press, 2010, 51-52. (in Chinese) 